\title{
COMMON FIXED POINT FOR COMPATIBLE MAPPINGS OF TYPE $(\alpha)$ ON INTUITIONISTIC FUZZY METRIC SPACE WITH IMPLICIT RELATIONS
}

\author{
JONG SEO PARK
}

\begin{abstract}
In this paper, we will establish common fixed point for compatible mappings of type $(\alpha)$ for four self mappings defined on intuitionistic fuzzy metric space with implicit relations.
\end{abstract}

\section{Introduction}

Several authors([4], [5]) have introduced the basic concepts on fuzzy metric spaces and fuzzy topological spaces induced by fuzzy metrics with different ways. Grabiec[2] obtained the Banach contraction principle in setting of fuzzy metric spaces. Also, I. Altun and D. Turkoglu[1] proved some fixed theorems using implicit relations in fuzzy metric spaces.

Recently, Park et.al.[11] defined the intuitionistic fuzzy metric space, and Park et.al.[7] proved a fixed point theorem of Banach for the contractive mapping of a complete intuitionistic fuzzy metric space, and Park and Kim[10] established common fixed point theorem for four self maps in intuitionistic fuzzy metric space.

In this paper, we will obtain a unique common fixed point theorem for compatible mappings of type $(\alpha)$ defined on intuitionistic fuzzy metric space under implicit relations.

\section{Preliminaries}

We will give some definitions, properties of the intuitionistic fuzzy metric space $X$ as following :

Received October 11, 2010. Accepted November 15, 2010.

2000 Mathematics Subject Classification. 46S40, 47H10, 54H25 .

Key words and phrases: common fixed point theorem, compatible mapping of type $(\alpha)$, implicit relation. 
Let us recall (see [12]) that a continuous $t$-norm is a binary operation $*:[0,1] \times[0,1] \rightarrow[0,1]$ which satisfies the following conditions:(a)* is commutative and associative; (b) $*$ is continuous; (c) $a * 1=a$ for all $a \in[0,1] ;(\mathrm{d}) a * b \leq c * d$ whenever $a \leq c$ and $b \leq d(a, b, c, d \in[0,1])$.

Similarly, a continuous $t$-conorm is a binary operation $\diamond:[0,1] \times$ $[0,1] \rightarrow[0,1]$ which satisfies the following conditions: $(\mathrm{a}) \diamond$ is commutative and associative; (b) $\diamond$ is continuous; (c) $a \diamond 0=a$ for all $a \in[0,1]$; (d) $a \diamond b \geq c \diamond d$ whenever $a \leq c$ and $b \leq d(a, b, c, d \in[0,1])$.

Definition 2.1. ([6]) The 5 -tuple $(X, M, N, *, \diamond)$ is said to be an intuitionistic fuzzy metric space if $X$ is an arbitrary set, $*$ is a continuous $t$-norm, $\diamond$ is a continuous $t$-conorm and $M, N$ are fuzzy sets on $X^{2} \times$ $(0, \infty)$ satisfying the following conditions; for all $x, y, z \in X$, such that
(a) $M(x, y, t)>0$,
(b) $M(x, y, t)=1 \Longleftrightarrow x=y$,
(c) $M(x, y, t)=M(y, x, t)$,
(d) $M(x, y, t) * M(y, z, s) \leq M(x, z, t+s)$,
(e) $M(x, y, \cdot):(0, \infty) \rightarrow(0,1]$ is continuous,
(f) $N(x, y, t)>0$,
(g) $N(x, y, t)=0 \Longleftrightarrow x=y$,
(h) $N(x, y, t)=N(y, x, t)$,
(i) $N(x, y, t) \diamond N(y, z, s) \geq N(x, z, t+s)$,
(j) $N(x, y, \cdot):(0, \infty) \rightarrow(0,1]$ is continuous.

Note that $(M, N)$ is called an intuitionistic fuzzy metric on $X$. The functions $M(x, y, t)$ and $N(x, y, t)$ denote the degree of nearness and the degree of non-nearness between $x$ and $y$ with respect to $t$, respectively.

Lemma 2.2. ([8]) For all $x, y \in X, M(x, y, \cdot)$ is nondecreasing on $(0, \infty)$ and $N(x, y, \cdot)$ is nonincreasing on $(0, \infty)$.

Definition 2.3. ([10]) Let $X$ be an intuitionistic fuzzy metric space.

(a) $\left\{x_{n}\right\}$ is said to be convergent to a point $x \in X$ if, for any $0<$ $\epsilon<1$ and $t>0$, there exists $n_{0} \in N$ such that $M\left(x_{n}, x, t\right)>1-\epsilon$, $N\left(x_{n}, x, t\right)<\epsilon$ for all $n \geq n_{0}$.

(b) $\left\{x_{n}\right\}$ is called a Cauchy sequence if for any $0<\epsilon<1$ and $t>0$, there exists $n_{0} \in N$ such that $M\left(x_{n}, x_{m}, t\right)>1-\epsilon, N\left(x_{n}, x_{m}, t\right)<\epsilon$ for all $m, n \geq n_{0}$.

(c) $X$ is complete if every Cauchy sequence converges in $X$.

Lemma 2.4. ([9])Let $X$ be an intuitionistic fuzzy metric space. If there exists a number $k \in(0,1)$ such that for all $x, y \in X$ and $t>0$,

$$
M(x, y, k t) \geq M(x, y, t), N(x, y, k t) \leq N(x, y, t),
$$


then $x=y$.

Definition 2.5. ([9])Let $A, B$ be mappings from intuitionistic fuzzy metric space $X$ into itself. The mappings are said to be compatible of type $(\alpha)$ if

$$
\begin{aligned}
& \lim _{n \rightarrow \infty} M\left(A B x_{n}, B B x_{n}, t\right)=1 \text { and } \lim _{n \rightarrow \infty} M\left(B A x_{n}, A A x_{n}, t\right)=1, \\
& \lim _{n \rightarrow \infty} N\left(A B x_{n}, B B x_{n}, t\right)=0 \text { and } \lim _{n \rightarrow \infty} N\left(B A x_{n}, A A x_{n}, t\right)=0
\end{aligned}
$$

for all $t>0$, whenever $\left\{x_{n}\right\} \subset X$ such that $\lim _{n \rightarrow \infty} A x_{n}=\lim _{n \rightarrow \infty} B x_{n}$ $=x$ for some $x \in X$.

Implicit relations on fuzzy metric spaces have been used in many $\operatorname{articles}\left([1],[3]\right.$ etc). Let $\Psi=\left\{\phi_{M}, \psi_{N}\right\}, I=[0,1], *, \diamond$ be a continuous t-norm, t-conorm and $\phi_{M}, \psi_{N}: I^{6} \rightarrow R$ be continuous functions. Now, we consider the following conditions:

(I) $\phi_{M}$ is decreasing and $\psi_{N}$ is increasing in sixth variables.

(II)If, for some $k \in(0,1)$, we have

$$
\begin{aligned}
\phi_{M}\left(u(k t), v(t), v(t), u(t), 1, u\left(\frac{t}{2}\right) * v\left(\frac{t}{2}\right)\right) & \geq 1, \\
\psi_{N}\left(x(k t), y(t), y(t), x(t), 0, x\left(\frac{t}{2}\right) \diamond y\left(\frac{t}{2}\right)\right) & \leq 1 \\
\text { or } \quad \phi_{M}\left(u(k t), v(t), u(t), v(t), u\left(\frac{t}{2}\right) * v\left(\frac{t}{2}\right), 1\right) & \geq 1, \\
\psi_{N}\left(x(k t), y(t), x(t), y(t), x\left(\frac{t}{2}\right) \diamond y\left(\frac{t}{2}\right), 0\right) & \leq 1
\end{aligned}
$$

for any fixed $t>0$, any nondecreasing functions $u, v:(0, \infty) \rightarrow I$ with $0<u(t), v(t) \leq 1$, and any nonincreasing functions $x, y:(0, \infty) \rightarrow I$ with $0<x(t), y(t) \leq 1$, then there exists $h \in(0,1)$ with $u(h t) \geq v(t) *$ $u(t), x(h t) \leq y(t) \diamond x(t)$.

(III)If, for some $k \in(0,1)$, we have $\phi_{M}(u(k t), u(t), 1,1, u(t), u(t)) \geq 1$ for any fixed $t>0$ and any nondecreasing function $u:(0, \infty) \rightarrow I$, then $u(k t) \geq u(t)$. Also, if, for some $k \in(0,1)$, we have $\psi_{N}(x(k t), x(t), 0,0, x(t)$, $x(t)) \leq 1$ for any fixed $t>0$ and any nonincreasing function $x:(0, \infty) \rightarrow$ $I$, then $x(k t) \leq x(t)$.

Example 2.6. Let $a * b=\min \{a, b\}$ and $a \diamond b=\max \{a, b\}$,

$$
\phi_{M}\left(u_{1}, \cdots, u_{6}\right)=\frac{u_{1}}{\min \left\{u_{2}, \cdots, u_{6}\right\}}, \quad \psi_{N}\left(x_{1}, \cdots, x_{6}\right)=\frac{x_{1}}{\max \left\{x_{2}, \cdots, x_{6}\right\}} .
$$


Also, let $t>0,0<u(t), v(t), x(t), y(t) \leq 1, k \in\left(0, \frac{1}{2}\right)$ where $u, v$ : $[0, \infty) \rightarrow I$ are nondecreasing functions and $x, y:[0, \infty) \rightarrow I$ are nonincreasing functions. Now, suppose that

$$
\begin{aligned}
& \phi_{M}\left(u(k t), v(t), v(t), u(t), 1, u\left(\frac{t}{2}\right) * v\left(\frac{t}{2}\right)\right) \geq 1, \\
& \psi_{N}\left(x(k t), y(t), y(t), x(t), 0, x\left(\frac{t}{2}\right) \diamond y\left(\frac{t}{2}\right)\right) \leq 1,
\end{aligned}
$$

then

$$
\begin{aligned}
& \phi_{M}\left(u(k t), v(t), v(t), u(t), 1, u\left(\frac{t}{2}\right) * v\left(\frac{t}{2}\right)\right)=\frac{u(k t)}{\min \left\{u\left(\frac{t}{2}\right), v\left(\frac{t}{2}\right)\right)} \geq 1, \\
& \psi_{N}\left(x(k t), y(t), y(t), x(t), 0, x\left(\frac{t}{2}\right) \diamond y\left(\frac{t}{2}\right)\right)=\frac{x(k t)}{\max \left\{x\left(\frac{t}{2}\right), y\left(\frac{t}{2}\right)\right)} \leq 1 .
\end{aligned}
$$

Thus, $u(h t) \geq v(t) * u(t), x(h t) \leq y(t) \diamond x(t)$. Suppose that $t>0$ is fixed, $u:(0, \infty) \rightarrow I$ is a nondecreasing, $x:(0, \infty) \rightarrow I$ nonincreasing function and

$$
\begin{aligned}
& \phi_{M}(u(k t), u(t), 1,1, u(t), u(t))=\frac{u(k t)}{u(t)} \geq 1, \\
& \psi_{N}(x(k t), x(t), 0,0, x(t), x(t))=\frac{x(k t)}{x(t)} \leq 1
\end{aligned}
$$

for $k \in(0,1)$. Then we have $u(k t) \geq u(t)$ and $x(k t) \leq x(t)$. Hence $\phi_{M}, \psi_{N} \in \Psi$.

\section{Main Results}

Now, we will prove some common fixed point theorem for four mappings on complete intuitionistic fuzzy metric space as follows:

Theorem 3.1. Let $(X, M, N, *, \diamond)$ be a complete intuitionistic fuzzy metric space with $a * b=\min \{a, b\}, a \diamond b=\max \{a, b\}$ for all $a, b \in I$ and $A, B, S$ and $T$ be mappings from $X$ into itself satisfying the conditions:
(a) $S(X) \subseteq B(X)$ and $T(X) \subseteq A(X)$,
(b)one of the mappings $A, B, S, T$ is continuous,
(c) $A$ and $S$ as well as $B$ and $T$ are compatible of type $(\alpha)$ 
(d) there exist $k \in(0,1)$ and $\phi_{M}, \psi_{N} \in \Psi$ such that

$$
\begin{aligned}
& \phi_{M}\left(\begin{array}{l}
M(S x, T y, k t), M(A x, B y, t), M(S x, A x, t), \\
M(T y, B y, t), M(S x, B y, t), M(T y, A x, t)
\end{array}\right) \geq 1, \\
& \psi_{N}\left(\begin{array}{l}
N(S x, T y, k t), N(A x, B y, t), N(S x, A x, t), \\
N(T y, B y, t), N(S x, B y, t), N(T y, A x, t)
\end{array}\right) \leq 1,
\end{aligned}
$$

for all $x, y \in X$ and $t>0$.

Then $A, B, S$ and $T$ have a unique common fixed point in $X$.

Proof. Let $x_{0}$ be an arbitrary point of $X$. From (a), we can construct a sequence a sequence $\left\{y_{n}\right\} \subset X$ as follows: $y_{2 n+1}=S x_{2 n}=B x_{2 n+1}$ and $y_{2 n+2}=T x_{2 n+1}=A x_{2 n+2}$ for all $n=0,1,2, \cdots$. Then, by (d), we have, for any $t>0$,

$$
\begin{aligned}
& \phi_{M}\left(\begin{array}{l}
M\left(S x_{2 n}, T x_{2 n+1}, k t\right), M\left(A x_{2 n}, B x_{2 n+1}, t\right), M\left(S x_{2 n}, A x_{2 n}, t\right), \\
M\left(T x_{2 n+1}, B x_{2 n+1}, t\right), M\left(S x_{2 n}, B x_{2 n+1}, t\right), M\left(T x_{2 n+1}, A x_{2 n}, t\right)
\end{array}\right) \geq 1, \\
& \psi_{N}\left(\begin{array}{l}
N\left(S x_{2 n}, T x_{2 n+1}, k t\right), N\left(A x_{2 n}, B x_{2 n+1}, t\right), N\left(S x_{2 n}, A x_{2 n}, t\right), \\
N\left(T x_{2 n+1}, B x_{2 n+1}, t\right), N\left(S x_{2 n}, B x_{2 n+1}, t\right), N\left(T x_{2 n+1}, A x_{2 n}, t\right)
\end{array}\right) \leq 1,
\end{aligned}
$$

and so

$$
\begin{gathered}
\phi_{M}\left(\begin{array}{l}
M\left(S x_{2 n}, T x_{2 n+1}, k t\right), M\left(T x_{2 n-1}, S x_{2 n}, t\right), M\left(S x_{2 n}, T x_{2 n-1}, t\right), \\
M\left(T x_{2 n+1}, S x_{2 n}, t\right), 1, M\left(T x_{2 n+1}, S x_{2 n}, \frac{t}{2}\right) * M\left(S x_{2 n}, T x_{2 n-1}, \frac{t}{2}\right)
\end{array}\right) \geq 1, \\
\psi_{N}\left(\begin{array}{l}
N\left(S x_{2 n}, T x_{2 n+1}, k t\right), N\left(T x_{2 n-1}, S x_{2 n}, t\right), N\left(S x_{2 n}, T x_{2 n-1}, t\right), \\
N\left(T x_{2 n+1}, S x_{2 n}, t\right), 0, N\left(T x_{2 n+1}, S x_{2 n}, \frac{t}{2}\right) \diamond N\left(S x_{2 n}, T x_{2 n-1}, \frac{t}{2}\right)
\end{array}\right) \leq 1,
\end{gathered}
$$

By (II), we have

$$
\begin{aligned}
& M\left(S x_{2 n}, T x_{2 n+1}, h t\right) \geq M\left(S x_{2 n}, T x_{2 n-1}, t\right) * M\left(S x_{2 n}, T x_{2 n+1}, t\right), \\
& N\left(S x_{2 n}, T x_{2 n+1}, h t\right) \leq N\left(S x_{2 n}, T x_{2 n-1}, t\right) \diamond N\left(S x_{2 n}, T x_{2 n+1}, t\right)
\end{aligned}
$$

and so,

$$
\begin{aligned}
& M\left(y_{2 n+1}, y_{2 n+2}, h t\right) \geq M\left(y_{2 n+1}, y_{2 n}, t\right) * M\left(y_{2 n+1}, y_{2 n+2}, t\right), \\
& N\left(y_{2 n+1}, y_{2 n+2}, h t\right) \leq N\left(y_{2 n+1}, y_{2 n}, t\right) \diamond N\left(y_{2 n+1}, y_{2 n+2}, t\right)
\end{aligned}
$$

which implies that

$M\left(y_{2 n+1}, y_{2 n+2}, h t\right) \geq M\left(y_{2 n+1}, y_{2 n}, t\right), \quad N\left(y_{2 n+1}, y_{2 n+2}, h t\right) \leq N\left(y_{2 n+1}, y_{2 n}, t\right)$

Also, by (II), we have

$M\left(y_{2 n+1}, y_{2 n}, h t\right) \geq M\left(y_{2 n}, y_{2 n-1}, t\right), \quad N\left(y_{2 n+1}, y_{2 n}, h t\right) \leq N\left(y_{2 n}, y_{2 n-1}, t\right)$.

Therefore, we have, for all $m=1,2, \cdots$, and $t>0$,

$M\left(y_{m+1}, y_{m+2}, h t\right) \geq M\left(y_{m}, y_{m+1}, t\right), \quad N\left(y_{m+1}, y_{m+2}, h t\right) \leq N\left(y_{m}, y_{m+1}, t\right)$. 
To prove that $\left\{y_{n}\right\}$ is a Cauchy sequence. First, we show that, for any $0<\lambda<1$ and $t>0$,

(1) $\quad M\left(y_{n+1}, y_{n+m+1}, t\right)>1-\lambda, \quad N\left(y_{n+1}, y_{n+m+1}, t\right)<\lambda$

for all $n \geq n_{0}$ and $m \in N$. Inductively, by above equation, we have, as $n \rightarrow \infty$

$$
\begin{aligned}
& M\left(y_{n+1}, y_{n+2}, t\right) \geq M\left(y_{n}, y_{n+1}, \frac{t}{h} \geq \cdots \cdots \geq M\left(y_{1}, y_{2}, \frac{t}{h^{n}}\right) \rightarrow 1,\right. \\
& N\left(y_{n+1}, y_{n+2}, t\right) \leq N\left(y_{n}, y_{n+1}, \frac{t}{h} \leq \cdots \cdots \leq N\left(y_{1}, y_{2}, \frac{t}{h^{n}}\right) \rightarrow 0 .\right.
\end{aligned}
$$

Hence, we can choose $n_{0} \in N$ such that for all $n \geq n_{0}$,

$$
M\left(y_{n+1}, y_{n+2}, t\right)>1-\lambda, \quad N\left(y_{n+1}, y_{n+2}, t\right)<\lambda .
$$

Thus (3.1) is true for $m=1$. Suppose that (3.1) is true for some $m \in N$. Then, for $m+1 \in N$, we have

$$
\begin{aligned}
& M\left(y_{n+1}, y_{n+m+2}, t\right) \geq M\left(y_{n+1}, y_{n+m+1}, \frac{t}{2}\right) * M\left(y_{n+m+1}, y_{n+m+2}, \frac{t}{2}\right) \geq 1-\lambda, \\
& N\left(y_{n+1}, y_{n+m+2}, t\right) \leq N\left(y_{n+1}, y_{n+m+1}, \frac{t}{2}\right) \diamond N\left(y_{n+m+1}, y_{n+m+2}, \frac{t}{2}\right) \leq \lambda .
\end{aligned}
$$

Hence (3.1) is true for $m+1 \in N$. Therefore $\left\{y_{n}\right\}$ is Cauchy sequence in $X$. Since $X$ is complete, $\left\{y_{n}\right\}$ converges to a point $x \in X$. Since $\left\{A x_{2 n+2}\right\},\left\{B x_{2 n+1}\right\},\left\{S x_{2 n}\right\}$ and $\left\{T x_{2 n+1}\right\} \subset\left\{y_{n}\right\}$, we have

$$
\lim _{n \rightarrow \infty} A x_{2 n+2}=\lim _{n \rightarrow \infty} B x_{2 n+1}=\lim _{n \rightarrow \infty} S x_{2 n}=\lim _{n \rightarrow \infty} T x_{2 n+1}=x .
$$

Now, suppose that $A$ is continuous. Then $\lim _{n \rightarrow \infty} A S x_{2 n}=A x$. Also, since $A, S$ are compatible of type $(\alpha), \lim _{n \rightarrow \infty} S A x_{2 n}=A x$. Using (d), we have, for any $t>0$,

$$
\begin{aligned}
& \phi_{M}\left(\begin{array}{l}
M\left(S A x_{2 n}, T x_{2 n+1}, k t\right), M\left(A A x_{2 n}, B x_{2 n+1}, t\right), M\left(S A x_{2 n}, A A x_{2 n}, t\right), \\
M\left(T x_{2 n+1}, B x_{2 n+1}, t\right), M\left(S A x_{2 n}, B x_{2 n+1}, t\right), M\left(T x_{2 n+1}, A A x_{2 n}, t\right)
\end{array}\right) \geq 1, \\
& \psi_{N}\left(\begin{array}{l}
N\left(S A x_{2 n}, T x_{2 n+1}, k t\right), N\left(A A x_{2 n}, B x_{2 n+1}, t\right), N\left(S A x_{2 n}, A A x_{2 n}, t\right), \\
N\left(T x_{2 n+1}, B x_{2 n+1}, t\right), N\left(S A x_{2 n}, B x_{2 n+1}, t\right), N\left(T x_{2 n+1}, A A x_{2 n}, t\right)
\end{array}\right) \leq 1
\end{aligned}
$$

and then letting $n \rightarrow \infty, \phi_{M}, \psi_{N}$ are continuous, we have

$$
\begin{aligned}
& \phi_{M}\left(\begin{array}{l}
M(A x, x, k t), M(A x, x, t), M(A x, x, t), \\
M(x, x, t), M(A x, x, t), M(x, x, t)
\end{array}\right) \geq 1, \\
& \psi_{N}\left(\begin{array}{l}
N(A x, x, k t), N(A x, x, t), N(A x, x, t), \\
N(x, x, t), N(A x, x, t), N(x, x, t)
\end{array}\right) \leq 1 .
\end{aligned}
$$

Therefore, by (III), we have

$$
M(A x, x, k t) \geq M(A x, x, t), \quad N(A x, x, k t) \leq N(A x, x, t) .
$$


Hence $A x=x$ from Lemma 2.4. Also, we have, by (d),

$$
\begin{aligned}
& \phi_{M}\left(\begin{array}{l}
M\left(S x, T x_{2 n+1}, k t\right), M\left(A x, B x_{2 n+1}, t\right), M(A x, S x, t), \\
M\left(T x_{2 n+1}, B x_{2 n+1}, t\right), M\left(S x, B x_{2 n+1}, t\right), M\left(T x_{2 n+1}, A x, t\right)
\end{array}\right) \geq 1, \\
& \psi_{N}\left(\begin{array}{l}
N\left(S x, T x_{2 n+1}, k t\right), N\left(A x, B x_{2 n+1}, t\right), N(A x, S x, t), \\
N\left(T x_{2 n+1}, B x_{2 n+1}, t\right), N\left(S x, B x_{2 n+1}, t\right), N\left(T x_{2 n+1}, A x, t\right)
\end{array}\right) \leq 1
\end{aligned}
$$

and, let $n \rightarrow \infty$, we get

$$
\begin{aligned}
& \phi_{M}\left(\begin{array}{l}
M(S x, x, k t), 1, M(x, S x, t), \\
1, M(S x, x, t), 1
\end{array}\right) \geq 1, \\
& \psi_{N}\left(\begin{array}{l}
N(S x, x, k t), 0, N(x, S x, t), \\
0, N(S x, x, t), 0
\end{array}\right) \leq 1 .
\end{aligned}
$$

On the other hand, since

$$
\begin{aligned}
& M(S x, x, t) \geq M\left(S x, x, \frac{t}{2}\right)=M\left(S x, x, \frac{t}{2}\right) * 1, \\
& N(S x, x, t) \leq N\left(S x, x, \frac{t}{2}\right)=N\left(S x, x, \frac{t}{2}\right) \diamond 0,
\end{aligned}
$$

$\phi_{M}$ is nonincreasing and $\psi_{N}$ is nondecreasing in the fifth variable, we have, for any $t>0$,

$$
\begin{aligned}
& \phi_{M}\left(\begin{array}{l}
M(S x, x, k t), 1, M(x, S x, t), \\
1, M(S x, x, t) * 1,1
\end{array}\right) \geq 1, \\
& \psi_{N}\left(\begin{array}{l}
N(S x, x, k t), 0, N(x, S x, t), \\
0, N(S x, x, t) \diamond 0,0
\end{array}\right) \leq 1
\end{aligned}
$$

which implies that $S x=x$. Since $S(X) \subseteq B(X)$, there exists a point $y \in X$ such that $B y=x$. Using (d), we have

$$
\begin{aligned}
& \phi_{M}\left(\begin{array}{l}
M(S x, T y, k t), M(A x, B y, t), M(S x, A x, t), \\
M(T y, B y, t), M(S x, B y, t), M(T y, A x, t)
\end{array}\right) \\
= & \phi_{M}\left(\begin{array}{l}
M(x, T y, k t), 1,1, \\
M(T y, x, t), 1, M(T y, x, t)
\end{array}\right) \geq 1, \\
& \psi_{N}\left(\begin{array}{l}
N(S x, T y, k t), N(A x, B y, t), N(S x, A x, t), \\
N(T y, B y, t), N(S x, B y, t), N(T y, A x, t)
\end{array}\right) \\
= & \psi_{N}\left(\begin{array}{l}
N(x, T y, k t), 0,0, \\
N(T y, x, t), 0, N(T y, x, t)
\end{array}\right) \leq 1
\end{aligned}
$$

which implies that $x=T y$. Since $B y=T y=x$ and $B, T$ are compatible of type $(\alpha)$, we have $T T y=B T y$. Hence $T x=T T y=B T y=B x$. 
Therefore, from (d), we have, for any $t>0$,

$$
\begin{aligned}
\phi_{M}\left(\begin{array}{l}
M(S x, T x, k t), M(A x, B x, t), M(S x, A x, t), \\
M(T x, B x, t), M(S x, B x, t), M(T x, A x, t)
\end{array}\right) \\
=\phi_{M}\left(\begin{array}{l}
M(x, T x, k t), M(x, T x, t), 1, \\
1, M(x, T x, t), 1, M(x, T x, t)
\end{array}\right) \geq 1, \\
\psi_{N}\left(\begin{array}{l}
N(S x, T x, k t), N(A x, B x, t), N(S x, A x, t), \\
N(T x, B x, t), N(S x, B x, t), N(T x, A x, t)
\end{array}\right) \\
=\psi_{N}\left(\begin{array}{l}
N(x, T x, k t), N(x, T x, t), 0, \\
0, N(x, T x, t), 0, N(x, T x, t)
\end{array}\right) \leq 1 .
\end{aligned}
$$

From (III), we have

$$
M(x, T x, k t) \geq M(x, T x, t), \quad N(x, T x, k t) \leq N(x, T x, t) .
$$

Therefore, we have $x=T x=B x$. Hence $X$ is a common fixed point of $A, B, S$ and $T$. The same result holds if we assume that $B$ is continuous insead of $A$.

Now, suppose that $S$ is continuous. Then $\lim _{n \rightarrow \infty} S A x_{2 n}=S x$. Since $A, S$ are compatible of type $(\alpha), \lim _{n \rightarrow \infty} A S x_{2 n}=S x$. Using (d), we have for any $t>0$,

$$
\begin{aligned}
& \phi_{M}\left(\begin{array}{l}
M\left(S S x_{2 n}, T x_{2 n+1}, k t\right), M\left(A S x_{2 n}, B x_{2 n+1}, t\right), M\left(S S x_{2 n}, A S x_{2 n}, t\right), \\
M\left(T x_{2 n+1}, B x_{2 n+1}, t\right), M\left(S S x_{2 n}, B x_{2 n+1}, t\right), M\left(T x_{2 n+1}, A S x_{2 n}, t\right)
\end{array}\right) \geq 1, \\
& \psi_{N}\left(\begin{array}{l}
N\left(S S x_{2 n}, T x_{2 n+1}, k t\right), N\left(A S x_{2 n}, B x_{2 n+1}, t\right), N\left(S S x_{2 n}, A S x_{2 n}, t\right), \\
N\left(T x_{2 n+1}, B x_{2 n+1}, t\right), N\left(S S x_{2 n}, B x_{2 n+1}, t\right), N\left(T x_{2 n+1}, A S x_{2 n}, t\right)
\end{array}\right) \leq 1,
\end{aligned}
$$

and then by $n \rightarrow \infty$, since $\phi_{M}, \psi_{N} \in \Psi$ are continuous, we have

$$
\begin{aligned}
& \phi_{M}\left(\begin{array}{l}
M(S x, x, k t), M(S x, x, t), 1, \\
1, M(S x, x, t), M(S x, x, t)
\end{array}\right) \geq 1, \\
& \psi_{N}\left(\begin{array}{l}
N(S x, x, k t), N(S x, x, t), 0, \\
0, N(S x, x, t), N(S x, x, t)
\end{array}\right) \leq 1 .
\end{aligned}
$$

Thus, we have, from (III),

$$
M(S x, x, k t) \geq M(S x, x, t), \quad N(S x, x, k t) \leq N(S x, x, t) .
$$

Hence $S x=x$. Since $S(X) \subseteq B(X)$, there exists a point $z \in X$ such that $B z=x$. Using (d), we have

$$
\begin{aligned}
& \phi_{M}\left(\begin{array}{l}
M\left(S S x_{2 n}, T z, k t\right), M\left(A S x_{2 n}, B z, t\right), M\left(S S x_{2 n}, A S x_{2 n}, t\right), \\
M(T z, B z, t), M\left(S S x_{2 n}, B z, t\right), M\left(T z, A S x_{2 n}, t\right)
\end{array}\right) \geq 1, \\
& \psi_{N}\left(\begin{array}{l}
N\left(S S x_{2 n}, T z, k t\right), N\left(A S x_{2 n}, B z, t\right), N\left(S S x_{2 n}, A S x_{2 n}, t\right), \\
N(T z, B z, t), N\left(S S x_{2 n}, B z, t\right), N\left(T z, A S x_{2 n}, t\right)
\end{array}\right) \leq 1,
\end{aligned}
$$


letting $n \rightarrow \infty$, we get

$$
\begin{aligned}
& \phi_{M}\left(\begin{array}{l}
M(x, T z, k t), 1,1 \\
M(x, T z, t), 1, M(x, T z, t)
\end{array}\right) \geq 1, \\
& \psi_{N}\left(\begin{array}{l}
N(x, T z, k t), 0,0 \\
N(x, T z, t), 0, N(x, T z, t)
\end{array}\right) \leq 1
\end{aligned}
$$

which implies that $x=T z$. Since $B z=T z=x$ and $B, T$ are compatible of type $(\alpha)$, we have $T B z=B B z$ and so $T x=T B z=B B z=B x$. Thus, we have

$$
\begin{aligned}
& \phi_{M}\left(\begin{array}{l}
M\left(S x_{2 n}, T x, k t\right), M\left(A x_{2 n}, B x, t\right), M\left(S x_{2 n}, A x_{2 n}, t\right), \\
M(T x, B x, t), M\left(S x_{2 n}, B x, t\right), M\left(T x, A x_{2 n}, t\right)
\end{array}\right) \geq 1, \\
& \psi_{N}\left(\begin{array}{l}
N\left(S x_{2 n}, T x, k t\right), N\left(A x_{2 n}, B x, t\right), N\left(S x_{2 n}, A x_{2 n}, t\right), \\
N(T x, B x, t), N\left(S x_{2 n}, B x, t\right), N\left(T x, A x_{2 n}, t\right)
\end{array}\right) \leq 1,
\end{aligned}
$$

letting $n \rightarrow \infty$,

$$
\begin{gathered}
\phi_{M}\left(\begin{array}{l}
M(x, T x, k t), M(x, T x, t), 1 \\
1, M(x, T x, t), M(x, T x, t)
\end{array}\right) \geq 1 \\
\psi_{N}\left(\begin{array}{l}
N(x, T x, k t), N(x, T x, t), 0 \\
0, N(x, T x, t), N(x, T x, t)
\end{array}\right) \leq 1
\end{gathered}
$$

Thus, $x=T x=B x$. Since $T(X) \subseteq A(X)$, there exists $w \in X$ such that $A w=x$. Thus, from (d),

$$
\begin{aligned}
& \phi_{M}\left(\begin{array}{l}
M(S w, T x, k t), M(A w, B x, t), M(S w, A w, t), \\
M(T x, B x, t), M(S w, B x, t), M(T x, A w, t)
\end{array}\right) \\
=\phi_{M} & \left(\begin{array}{l}
M(S w, x, k t), 1, M(S w, x, t), \\
1, M(S w, x, t), 1
\end{array}\right) \geq 1, \\
& \psi_{N}\left(\begin{array}{l}
N(S w, T x, k t), N(A w, B x, t), N(S w, A w, t), \\
N(T x, B x, t), N(S w, B x, t), N(T x, A w, t)
\end{array}\right) \\
= & \psi_{N}\left(\begin{array}{l}
N(S w, x, k t), 0, N(S w, x, t), \\
0, N(S w, x, t), 0
\end{array}\right) \leq 1 .
\end{aligned}
$$

Hence we have $x=S w=A w$. Also, since $A, S$ are compatible of type $(\alpha), x=S x=S A w=A A w=A x$. Hence $x$ is a common fixed point of $A, B, S$ and $T$. The same result holds if we assume that $T$ is continuous instead of $S$. 
Finally, suppose that $A, B, S$ and $T$ have another common fixed point $u$. Then we have, for any $t>0$,

$$
\begin{aligned}
& \phi_{M}\left(\begin{array}{l}
M(S x, T u, k t), M(A x, B u, t), M(S x, A x, t), \\
M(T u, B u, t), M(S x, B u, t), M(T u, A x, t)
\end{array}\right) \\
= & \phi_{M}\left(\begin{array}{l}
M(x, u, k t), M(x, u, t), 1, \\
1, M(x, u, t), M(x, u, t)
\end{array}\right) \geq 1, \\
& \psi_{N}\left(\begin{array}{l}
N(S x, T u, k t), N(A x, B u, t), N(S x, A x, t), \\
N(T u, B u, t), N(S x, B u, t), N(T u, A x, t)
\end{array}\right) \\
= & \psi_{N}\left(\begin{array}{l}
N(x, u, k t), N(x, u, t), 0, \\
0, N(x, u, t), N(x, u, t)
\end{array}\right) \leq 1 .
\end{aligned}
$$

Therefore, from (III), $x=u$. This completes the proof.

\section{References}

[1] Altun I., Turkoglu D., 2008. Some fixed point theorems on fuzzy metric spaces with implicit relations. Commun. Korean Math. Soc. 23, 111-124.

[2] Grabiec, M., 1988. Fixed point in fuzzy metric spaces. Fuzzy Sets and Systems $27,385-389$.

[3] Imbad M., Kumar S., Khan M.S., 2002. Remarks on some fixed point theorems satisfying implicit relations. Rad. Math. 11, 135-143.

[4] Kramosil,J., Michalek J., 1975. Fuzzy metric and statistical metric spaces. Kybernetica 11, 326-334.

[5] Kaleva, O., Seikkala, S., 1984. On fuzzy metric spaces. Fuzzy Sets and Systems 12, 215-229

[6] Park, J.H., Park, J.S., Kwun, Y.C., 2006. A common fixed point theorem in the intuitionistic fuzzy metric space. Advances in Natural Comput. Data Mining(Proc. 2nd ICNC and 3rd FSKD), 293-300.

[7] Park, J.H., Park, J.S., Kwun, Y.C., 2007. Fixed point theorems in intuitionistic fuzzy metric space(I). JP J. fixed point Theory \& Appl. 2(1), 79-89.

[8] Park, J.S., Kim, S.Y., 1999. A fixed point theorem in a fuzzy metric space. F.J.M.S. 1(6), 927-934.

[9] Park, J.S., Park, J.H., Kwun, Y.C., 2008. On some results for five mappings using compatibility of type $(\alpha)$ in intuitionistic fuzzy metric space. International J. Fuzzy Logic Intelligent Systems 8(4), 299-305

[10] Park, J.S., Kim, S.Y., 2008. Common fixed point theorem and example in intuitionistic fuzzy metric space. J. Fuzzy Logic and Intelligent Systems 18(4), 524-529.

[11] Park, J.S., Kwun, Y.C., Park, J.H., 2005. A fixed point theorem in the intuitionistic fuzzy metric spaces. F.J.M.S. 16(2), 137-149.

[12] Schweizer, B., Sklar, A., 1960. Statistical metric spaces. Pacific J. Math. 10, 314-334. 
Department of Mathematics Education

Chinju National University of Education

Jinju 660-756, Korea

E-mail: parkjs@cue.ac.kr 